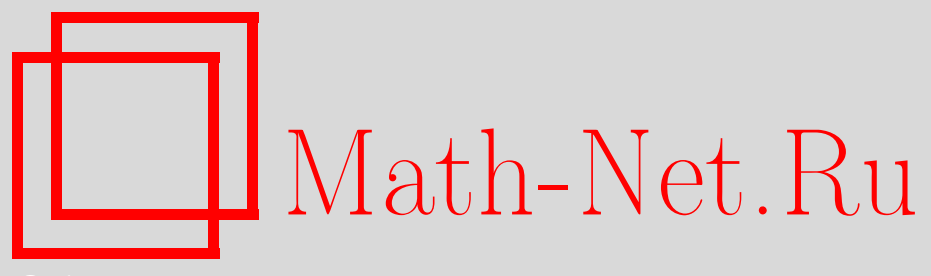

С. С. Галкин, В. В. Голышев, Квантовые когомологии грассманианов и круговые поля, УМН, 2006, том 61, выпуск $1,175-176$

DOI: https://doi.org/10.4213/rm1705

Использование Общероссийского математического портала Math-Net.Ru подразумевает, что вы прочитали и согласны с пользовательским соглашением http://www . mathnet.ru/rus/agreement

Параметры загрузки:

IP : 35.173 .219 .12

26 апреля 2023 г., 15:45:23

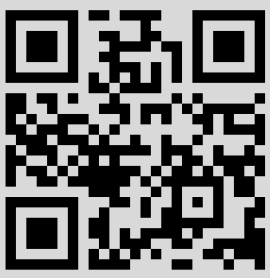




\section{Квантовые когомологии грассманианов и круговые поля}

\section{С. С. Галкин, В. В. Голышев}

Квантовые когомологии грассманианов [1]. Пусть $1 \leqslant l<N-$ пара целых чисел, $G=G(l, N)$ - грассманиан $l$-мерных подпространств в $N$-мерном векторном пространстве. Через $S$ обозначим тавтологическое подрасслоение на $G$. Обозначим через $\Omega_{\lambda}$ класс Шуберта в $H^{*}(G)$, соответствующий разбиению $\lambda$. Трехточечный коррелятор $\left\langle\Omega_{\lambda}, \Omega_{\mu}, \Omega_{\nu}\right\rangle_{d}$ определяется как число рациональных кривых степени $d$, пересекающих общих представителей этих классов. Алгебра $Q H^{*}(G, \mathbb{Z})$ является $\mathbb{Z}[q]$-модулем, изоморфным $H^{*}(G, Z)_{\mathbb{Z}} \otimes \mathbb{Z}[q]$ и снабженным следующей структурой умножения. Обозначим классы Шуберта в этой алгебре через $\sigma_{\lambda}$, так что $\sigma_{\lambda}=\Omega_{\lambda} \otimes 1$. Положим $\sigma_{\lambda} \cdot \sigma_{\mu}=\sum_{\nu, d \geqslant 0}\left\langle\sigma_{\lambda}, \sigma_{\mu}, \sigma_{\nu} \vee\right\rangle_{d} q^{d} \sigma_{\nu}$, где $\nu^{\vee}-$ разбиение, двойственное к $\nu$.

Пусть $\zeta$ - первообразный корень степени $N$ из $(-1)^{l+1}$.

Положим $K=\mathbb{Q}(\zeta), k=N-l$. Пусть $\Lambda-$ (градуированное) кольцо симметрических функций, $\Lambda=\mathbb{Z}\left[e_{1}, e_{2}, \ldots\right]$. Здесь $e_{i}$ есть $i$-я элементарная симметрическая функция. Пусть $h_{i}-i$-я полная симметрическая функция. Полагая $E(t)=\sum e_{i} t^{i}, H(t)=$ $\sum h_{i} t^{i}$, имеем $E(-t) H(t)=1$. Результат применения симметрической функции $\sigma$ к набору аргументов $x_{1}, \ldots, x_{n}$ будем обозначать $\sigma\left(x_{1}, \ldots, x_{n}\right)$.

Положим еще $\Lambda^{\prime}=\Lambda /\left(e_{l+1}, e_{l+2}, \ldots\right), \Lambda_{\mathbb{Q}}=\Lambda \otimes \mathbb{Q} ; \Lambda_{\mathbb{Q}}^{\prime}=\Lambda^{\prime} \otimes \mathbb{Q}$. Наконец, пусть $\Lambda_{K}^{\prime}=\Lambda_{\mathbb{Q}}^{\prime} \otimes_{\mathbb{Q}} K$.

Теорема 1 (Зиберт-Тиан [2]). Определим гомоморфизм колеи, $S T: \Lambda[q] \rightarrow Q H(G, \mathbb{Z})$ следуюшим образом: $S T(q)=1 \otimes q ; S T\left(e_{i}\right)=c_{i}\left(S^{*}\right) \otimes 1$. Тогда $S T$ - эпиморфизм и Ker $S T=\left(e_{l+1}, e_{l+2}, \ldots ; h_{N-l+1}, \ldots, h_{N-1}, h_{N}+(-1)^{l} q\right)$.

Пусть $Q H(G, \mathbb{Q})=Q H(G, \mathbb{Z}) \otimes \mathbb{Q}$ - кольцо рациональных квантовых когомологий. Через $Q H(G, \mathbb{Q}, 1)$ мы обозначаем специализацию кольца $Q H(G, \mathbb{Q})$ при $q=1$. Положим $I_{1}=\left(h_{N-l+1}, \ldots, h_{N-1}, h_{N}+(-1)^{l}\right)$, тогда, разумеется, $Q H(G, \mathbb{Q}, 1)=\Lambda_{\mathbb{Q}}^{\prime} / I_{1}$. Аналогично определим $K$-алгебру $Q H(G, K, 1)=Q H(G, \mathbb{Q}, 1) \otimes \mathbb{Q} K$ и идеал $I_{1}^{K}=$ $I_{1} \otimes K$.

Обозначим через $\theta_{1}, \ldots, \theta_{N}$ различные корни из 1 степени $N$. Пусть $J=j_{1} j_{2} \ldots j_{l}$ - мультииндекс, нумерующий $l$-ки различных корней степени $N$ из 1 , так что $j_{1} \leqslant$ $\ldots \leqslant j_{l}, j_{k} \in\{1, \ldots, N\}$. Мы будем писать $\theta_{J}$ вместо $\left\{\theta_{j_{1}}, \ldots, \theta_{j_{l}}\right\}$ и $\sigma\left(\zeta \theta_{J}\right)$ вместо $\sigma\left(\zeta \theta_{j_{1}}, \ldots, \zeta \theta_{j_{l}}\right)$. Положим $\theta_{\bar{J}}=\left\{\theta_{1}, \ldots, \theta_{N}\right\} \backslash\left\{\theta_{j_{1}}, \ldots, \theta_{j_{l}}\right\}$. Пусть $\phi_{J}: \Lambda_{K}^{\prime} \rightarrow K-$ гомоморфизм, определенный условием $\phi_{J}(\sigma)=\sigma\left(\zeta \theta_{J}\right)$. Обозначим $\operatorname{Ker} \phi_{J}$ через $I_{J}$. Положим $\phi=\bigoplus \phi_{J}$.

Лемма 2. Если $J \neq J^{\prime}$, mo $I_{J} \neq I_{J^{\prime}}$.

ДоказАтельство. В самом деле, если $K$-точки на $\mathbb{A}_{K}^{l}=\operatorname{Spec} \Lambda_{K}^{\prime}$, соответствующие идеалам $I_{J}$ и $I_{J^{\prime}}$, совпадают, то совпадают и их координаты, т.е. значения симметрических функций $\sigma_{1}, \ldots, \sigma_{l}$ на наборах $\theta_{J}$ и $\theta_{J^{\prime}}$. Значит, совпадают и сами наборы.

Лемма 3. Для фиксированного $J$ имеем вложение идеалов $I_{1}^{K} \subset I_{J}$.

Доказательство. В самом деле, $\left(\sum_{i=0}^{l} e_{i}\left(\theta_{J}\right)(-t)^{i}\right)\left(\sum_{i=0}^{k} e_{i}\left(\theta_{\bar{J}}\right)(-t)^{i}\right)=1-t^{N}$. С другой стороны, $\left(\sum_{i=0}^{l} e_{i}\left(\theta_{J}\right)(-t)^{i}\right)\left(\sum_{i=0}^{\infty} h_{i}\left(\theta_{J}\right) t^{i}\right)=1$. Значит,

$$
\begin{aligned}
\sum_{i=0}^{k} e_{i}\left(\theta_{\bar{J}}\right)(-t)^{i} & =\left(\sum_{i=0}^{l} e_{i}\left(\theta_{J}\right)(-t)^{i}\right)\left(\sum_{i=0}^{k} e_{i}\left(\theta_{\bar{J}}\right)(-t)^{i}\right)\left(\sum_{i=0}^{\infty} h_{i}\left(\theta_{J}\right) t^{i}\right) \\
& =\left(1-t^{N}\right) \sum_{i=0}^{\infty} h_{i}\left(\theta_{J}\right) t^{i} .
\end{aligned}
$$

Работа первого автора поддержана грантами РФФИ 04-01-00613 и 04-01-00702. 
Сопоставляя коэффициенты при $t^{i}$ слева и справа, находим $h_{i}\left(\theta_{J}\right)=0$ для $i=$ $k+1, \ldots, N-1$ и $h_{N}\left(\theta_{J}\right)-1=0$. Соответственно, $h_{i}\left(\zeta \theta_{J}\right)=0$ для $i=k+1, \ldots, N-1$ и $h_{N}\left(\zeta \theta_{J}\right)=\zeta^{N}=(-1)^{l+1}$.

Следствие 4. Имеем вложение идеалов $I_{1}^{K} \subset \operatorname{Ker} \phi$.

СлЕДСтвиЕ 5. Представление ф пропускается через гомоморбизм конечномерных коммутативных алгебр $\psi: Q H^{*}(G, K, 1) \rightarrow \bigoplus_{J} K$,

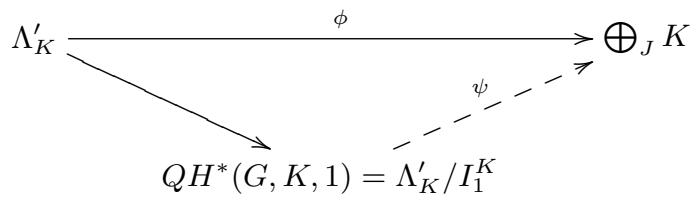

Теорема 6. Гомоморфизм $\psi$ является изоморфизмом.

ДокАзАтельство. Нам следует установить равенство $\operatorname{Ker} \phi=I_{1}^{K}$. По лемме 3 имеем вложение $I_{1}^{K} \subset \operatorname{Ker} \phi$. Ясно, что $\operatorname{Ker} \phi=\bigcap_{J} I_{J}$. Идеал $\operatorname{Ker} \phi$ является пересечением различных (по лемме 2) максимальных идеалов $I_{J}$. Как $K$-векторное пространство в кольце $\Lambda_{K}^{\prime}$, каждый из них имеет коразмерность, равную 1. Следовательно, их пересечение имеет коразмерность над $K$, равную их числу, т.е. $C_{N}^{l}$. С другой стороны, коразмерность идеала $I_{1}^{K}$ равна размерности факторкольца $\Lambda_{K}^{\prime} / I_{1}^{K}$. Согласно теореме Зиберта-Тиана, это кольцо изоморфно кольцу квантовых когомологий, которое является свободным модулем ранга $\operatorname{dim}_{K} H^{*}(G, K, 1)=C_{N}^{l}$ над $K$. Следовательно, идеалы $\operatorname{Ker} \phi$ и $I_{1}^{K}$ совпадают.

СледСтвие 7. Алгебра $\Lambda_{K}^{\prime} / I_{1}^{K}$ полупроста. Она является прямой суммой своих различных простых минимальных идеалов, каждый из которых изоморфен $K$.

СлЕДСтвиЕ 8. Алгебра $\Lambda_{\mathbb{Q}}^{\prime} / I_{1}$ полупроста. Собственные числа умножений на классы Шуберта лежат в $K$.

ГиПотезА 9. Пусть теперь $G$ - грассманиан максимальных изотропных плоскостей в четномерном пространстве, снабженном невырожденной квадратичной формой, или грассманиан лагранжевых плоскостей в пространстве с симплектической формой. Тогда собственные числа оператора умножения на класс дивизора, действующего в пространстве $Q H(G, \mathbb{Q}, 1)$, определены над круговым расширением $\mathbb{Q}$.

Пусть $G$ - обобщенный грассманиан, т.е. фактор (классической) простой группы по максимальной параболической подгруппе. Мы не ожидаем, вообще говоря, что собственные числа оператора умножения на класс дивизора будут определены над круговыми полями.

\section{Список литературы}

[1] A. Buch, Compositio Math., 137:2 (2003), 227-235. [2] B. Siebert, G. Tian, Asian J. Math., 1:4 (1997), 679-695.

\section{C. Галкин (S.S. Galkin)}

Математический институт им. В. А. Стеклова РАН

E-mail: galkin@mi.ras.ru

В. В. Голышев (V. V. Golyshev)

Математический институт им. В. А. Стеклова РАН
Представлено А. Г. Сергеевым Принято редколлегией 01.12.2005 